\title{
A!
}

This is an electronic reprint of the original article.

This reprint may differ from the original in pagination and typographic detail.

Harju, A.; Räsänen, E.; Saarikoski, H.; Puska, M.J.; Nieminen, R.M.; Niemelä, K.

\section{Broken symmetry in density-functional theory: Analysis and cure}

Published in:

Physical Review B

DOI:

10.1103/PhysRevB.69.153101

Published: 01/04/2004

Document Version

Publisher's PDF, also known as Version of record

Please cite the original version:

Harju, A., Räsänen, E., Saarikoski, H., Puska, M. J., Nieminen, R. M., \& Niemelä, K. (2004). Broken symmetry in density-functional theory: Analysis and cure. Physical Review B, 69(15), 1-4. [153101].

https://doi.org/10.1103/PhysRevB.69.153101

This material is protected by copyright and other intellectual property rights, and duplication or sale of all or part of any of the repository collections is not permitted, except that material may be duplicated by you for your research use or educational purposes in electronic or print form. You must obtain permission for any other use. Electronic or print copies may not be offered, whether for sale or otherwise to anyone who is not an authorised user. 


\title{
Broken symmetry in density-functional theory: Analysis and cure
}

\author{
A. Harju, E. Räsänen, H. Saarikoski, M. J. Puska, and R. M. Nieminen \\ Laboratory of Physics, Helsinki University of Technology, P.O. Box 1100, FIN-02015 HUT, Finland \\ K. Niemelä \\ Theoretical Physics, University of Oulu, P.O. Box 3000, FIN-90014 University of Oulu, Finland
}

(Received 26 January 2004; published 1 April 2004)

\begin{abstract}
We present a detailed analysis of the broken-symmetry mean-field solutions using a four-electron rectangular quantum dot as a model system. Comparisons of the density-functional theory predictions with the exact ones show that the symmetry-breaking results from the single-configuration wave function used in the mean-field approach. As a general cure we present a scheme that systematically incorporates several configurations into the density-functional theory and restores the symmetry. This cure is easily applicable to any density-functional approach.
\end{abstract}

DOI: 10.1103/PhysRevB.69.153101

PACS number(s): 73.21.La, 71.15.Mb, 71.10.Pm

\section{INTRODUCTION}

The nanoscale semiconductor systems are technically very promising for future components of microelectronic devices. From the theoretical point of view, quantum dot (QD) systems are valuable source of novel quantum effects. Many of these result from the fact that the electron-electron interaction and external magnetic field have greatly enhanced effects compared to atoms and molecules. This raises new challenges for the theoretical methods, and the validity of approximations in, e.g., mean-field approaches can be questioned. For this reason, QD systems serve as perfect test cases to develop the electronic structure methods, with the results still applicable to great variety of physical problems where mean-field approaches have been used.

In earlier studies, Hartree-Fock and especially densityfunctional theory (DFT) methods have shown to produce accurate results for various QD systems, even with small $N$. However, in the context of solutions with a broken spin symmetry, the validity of the mean-field approaches has been actively discussed in the literature. ${ }^{2}$ The spin-density wave (SDW) formation in QD's has been compared to similar phenomena found in isotropic metals, ${ }^{4}$ organic linear-chain compounds, ${ }^{5}$ atomic nuclei, ${ }^{6}$ and small fermion clusters. ${ }^{7}$ According to the Jahn-Teller theorem, any nonlinear molecular system in a degenerate electronic state becomes more stable by removing the degeneracy and thus lowering the symmetry and the total energy. A crucial difference between molecular and QD systems is, however, that as the nuclei in molecules are free to move and relax, the QD potential is external and fixed as it results from, e.g., metallic gates. Thus to lower the symmetry in QD, the spin densities must "relax" in an antiferromagnetic fashion to a SDW solution. This is claimed to reveal the electron correlations inherent in the true ground state. $^{2}$

In this paper, we analyze symmetry breaking in a twodimensional rectangular $\mathrm{QD}^{1}$ using both DFT and exact diagonalization (ED). We concentrate on the four-electron case, as it is the first particle number showing the general features of electronic structure seen also for larger particle numbers, such as the transitions between the two spin states
$S=0$ and 1 and the SDW solution predicted by DFT. We find that SDW clearly reflects the limitations of basic DFT to describe systems that have more than one major configuration in the ground-state wave function. There is a continuous interest for developing DFT methods for these kinds of systems. The main difficulty for DFT is the fact that these systems have ensemble- $v$-representable (E-VR) densities in contrast to the more common pure-state- $v$-representable (PVR) densities. ${ }^{3}$ As an interesting feature we see a continuous transition from an E-VR to a P-VR density as we deform our QD. Finally, we present a simple modification of DFT that is able to describe the multiconfigurational nature of the ground states.

\section{THE MODEL AND THE METHODS}

The generally used model Hamiltonian of an $N$-electron QD system can be written as

$$
H=\sum_{i=1}^{N}\left[-\frac{\hbar^{2}}{2 m^{*}} \nabla_{i}^{2}+V_{\text {ext }}\left(\mathbf{r}_{i}\right)\right]+\sum_{i<j}^{N} \frac{e^{2}}{\epsilon\left|\mathbf{r}_{i}-\mathbf{r}_{j}\right|},
$$

where we have used the effective-mass approximation to describe electrons moving in the $x y$ plane, surrounded by background material of GaAs with the effective electron mass $m^{*}=0.067 m_{e}$ and dielectric constant $\epsilon=12.4$. We use scaled atomic units, and energies are thus given in $\mathrm{Ha}^{*}$ $\approx 11.86 \mathrm{meV}$ and lengths in $a_{B}^{*} \approx 9.79 \mathrm{~nm}$. The external confinement in the $x y$ plane is described by an infinite hard-wall potential,

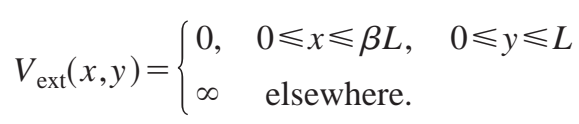

The deformation parameter $\beta$ defines the ratio between the side lengths of the rectangle. The area of the dot is fixed to be $\pi^{2}$. The single-particle eigenstates are sine functions in both directions, labeled with two quantum numbers $\left(n_{x}, n_{y}\right)$, and energies $E_{n_{x}, n_{y}}=\left(n_{x}^{2} / \beta+\beta n_{y}^{2}\right) / 2$. Figure 1 shows the three lowest eigenstates and the most important $S_{z}=0$ configurations of the four-electron QD. 

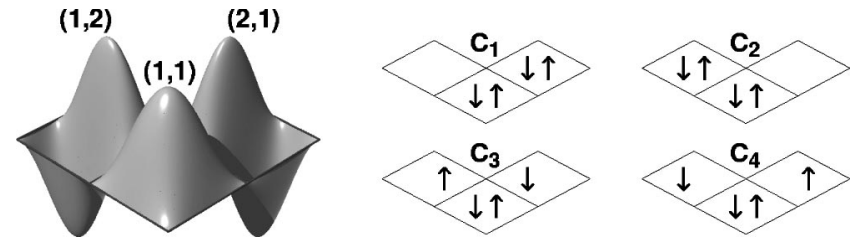

FIG. 1. Left panel: The three lowest single-particle states and their quantum numbers $\left(n_{x}, n_{y}\right)$. Right: Electron occupations for the four important $S_{z}=0$ configurations $C_{i}$.

We solve the electronic structure of QD using ED and DFT. In ED, the many-particle wave function is constructed as an expansion of the noninteracting eigenstates. The results approach the exact ones as more terms are added to the expansion. We use a basis of up to around 15,000 configurations. The interaction matrix elements are calculated numerically using Gaussian integration. In ED, all many-body quantum effects are taken into account in an exact fashion. In DFT, these are incorporated in a mean-field fashion as an effective potential. In the DFT method used, we allow different spin densities for up and down electrons. This is necessary for $S \neq 0$, and needed also for $S=0$ in order to find broken-symmetry solutions. More details of the DFT method and the numerical implementation can be found from Ref. 1, and references therein.

\section{RESULTS}

\section{A. Energies and densities for four-electron dot}

In Fig. 2 we present the DFT and ED energies of the rectangular quantum dot as a function of the deformation parameter $\beta$. For $\beta$ close to unity, the $S=1$ state is lower in energy than the $S=0$ state, in accordance with Hund's rule. In the case of the $S=1$ state, the DFT energies compare quite well with those obtained by ED: the deviation between the

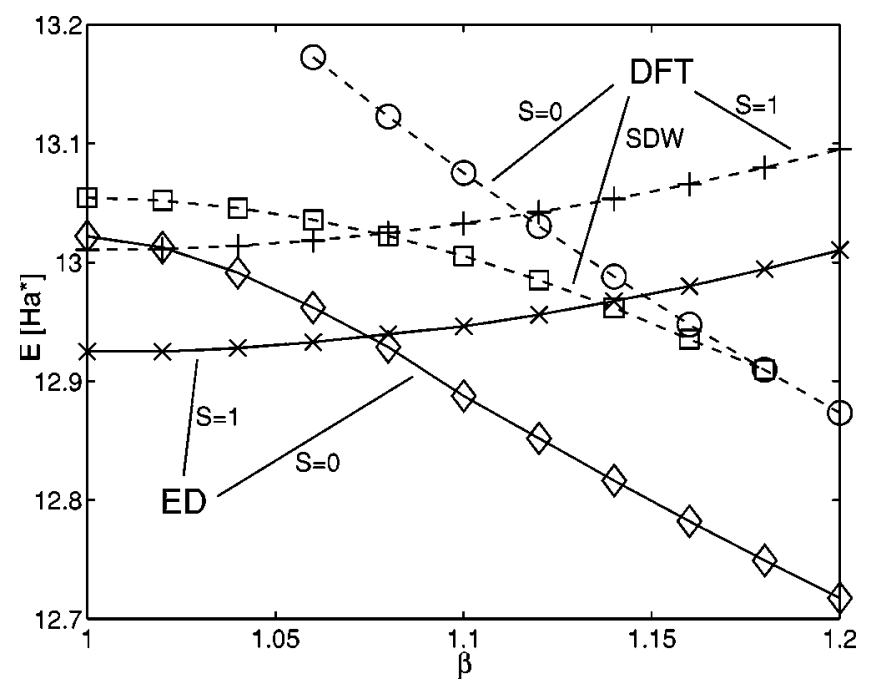

FIG. 2. Energy of the four-electron dot as a function of the axis ratio $\beta$. The solid lines present ED energies, we use crosses for $S$ $=1$ and diamonds for $S=0$, correspondingly. The dashed lines are DFT energies, pluses for $S=1$, boxes for the $S=0$ SDW solution, and circles for the symmetric $S=0$ energy.
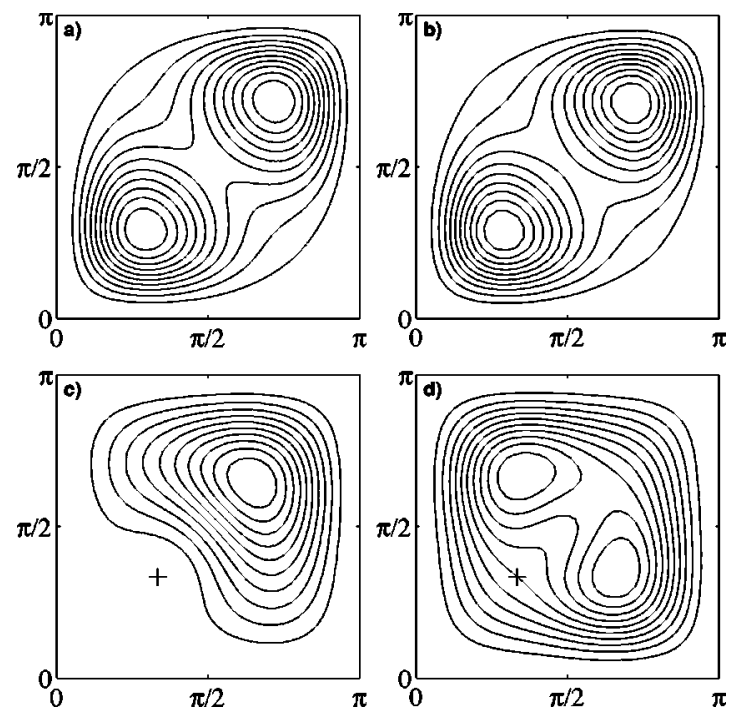

FIG. 3. (a) DFT spin density for the SDW solution. The density for the other spin type can be found through rotation by $90^{\circ}$ (b) ED spin density for the artificial sum of $S=0$ and $S=1$ states. (c) Conditional density from ED for the same spin type as the electron fixed at "+." (d) ED conditional density for opposite spins. The number of contour lines (drawn at uniform spacing) is fixed to 10 in each figure to ease comparisons. The SDW density is more similar to the unphysical ED density of (b) than the conditional densities.

two remains nearly constant for all values of $\beta$. Such a behavior is not seen in the $S=0$ results, for which we show two DFT energies: one with retained symmetry and another with a broken one. The broken-symmetry solution has a nonzero total spin density, corresponding to a SDW solution, see Fig. 3. The DFT calculation with the spin symmetry does not converge for smaller $\beta$ than those shown. This is due to the degeneracy in the system. Convergence can be achieved by use of fractional occupations. Comparing the DFT $S=0$ energies to the exact ones, one can see that, unlike for the $S$ $=1$ case, the error in DFT is not constant. The energy of the symmetry-restricted state grows linearly towards $\beta \rightarrow 1$ where the ED energy saturates. On the other hand, the SDW state has an energy that overcompensates the error in the symmetry-restricted energy. The energy of the SDW state is closer to the exact value than the energy of the propersymmetry state. One should note that the errors in DFT energies nearly cancel at the ground-state transition point, and the DFT prediction for it is very accurate.

It is claimed that the SDW spin densities reflect the internal structure of the system. ${ }^{2}$ To analyze this claim, we have plotted the SDW spin density of the DFT and ED conditional densities in Fig. 3. The conditional density is defined to be the electron density of the remaining three electrons as the coordinates of one of the electrons are fixed. In addition, we plot the ED spin density for the sum of the $S=0$ and $S=1$ states. One can see from the densities that there is a clear antiferromagnetic order in the system. Densities for parallel spins are localized in the opposite corners. Apart from this fact, the similarity of the conditional densities to the SDW density of Fig. 3 is marginal. However, the similarity of the SDW density to the unphysical mixture of the two-spin states 
is very clear. The only difference is that the DFT density is slightly more localized. One should note that this similarity of the SDW solution to a mixture of two different spin states is pointed out by Hirose and Wingreen using ED in restricted basis. $^{8}$

\section{B. Analysis of the broken symmetry}

To understand the electronic structure of the system and to analyze the problem associated with the SDW solution, it is enough to consider only the most important configurations in the ED solution, presented in Fig. 1 . The $S=1$ state, the ground state for small $\beta$, has three different $S_{z}$ states which are degenerate in energy. The one with $S_{z}=0$ consists of configurations $C_{3}$ and $C_{4}$ with equal weights for all values of $\beta$. The $S=0$ state is the ground state for large $\beta$, and it consists of the configurations $C_{1}$ and $C_{2}$. For $\beta=1$, these have equal weights, but for larger $\beta, C_{2}$ moves higher in energy and has a smaller weight in the exact wave function. For $\beta \approx 1.2, C_{1}$ is clearly the dominating configuration. One should note that at this value of $\beta$, proper symmetry is restored in the DFT solution. The most natural reason for the occurrence of the SDW solution is that the basic DFT is unable to take into account more than one important configuration for the construction of the Kohn-Sham orbitals and the resulting densities. In terms of the configurations, the DFT spin densities at $\beta=1.2$ correspond to $C_{1}$. For smaller $\beta$, however, the SDW spin densities can only be obtained by a linear combination of all four configurations. For $\beta=1$, this linear combination is equal to the unphysical mixture of two different spin states used for Fig. 3(b) above.

It is possible to analyze the broken-symmetry solution more generally by considering a mean-field-type singleconfiguration wave function for two up- and two down-spin electrons, occupying the orbitals $\psi_{0}$ and $\sin \left(\theta_{\sigma}\right) \psi_{1}$ $+\cos \left(\theta_{\sigma}\right) \psi_{2}$, where $\theta_{\sigma}$ contains the variational freedom for a spin type $\sigma$. Expanding this wave function results in four configurations similar to $\left\{C_{i}\right\}_{i=1}^{4}$ above. Assuming a further similarity to the QD case for $\beta=1$, one can write a Hamiltonian matrix of the four configurations as

$$
H=\left(\begin{array}{cccc}
E_{1} & \delta & 0 & 0 \\
\delta & E_{1} & 0 & 0 \\
0 & 0 & E_{0} & \delta \\
0 & 0 & \delta & E_{0}
\end{array}\right),
$$

where the configurations couple via the off-diagonal matrix element $\delta$ (taken to be real). The four exact energies are $E_{0} \pm \delta$ and $E_{1} \pm \delta$. One can set without loss of generality $E_{0}=0$ and $E_{1}=1$. The single-configuration energies have an interesting dependence on $\delta$, shown in Fig. 4. We present the energy as a function of the two variational angles $\theta$ for cases $\delta=0.2$ and 0.8. For small $\delta$, the second orbital for the minimum-energy solution is $\psi_{1}$ for one-spin type and $\psi_{2}$ for the other. For the case $\delta=0.8$, the minima are found with orbitals $\psi_{1}+\psi_{2}$ and $\psi_{1}-\psi_{2}$. The resulting total wave function of this case can easily be found to be a sum of the two exact wave functions (with energies $-\delta$ and $1-\delta$ ), and the
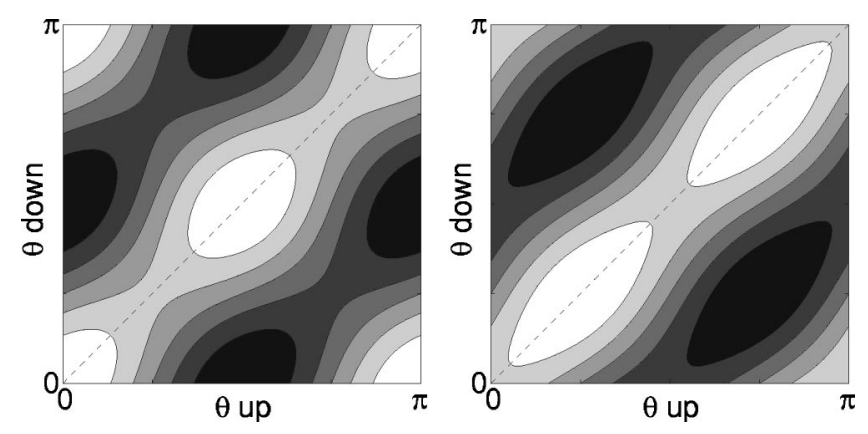

FIG. 4. Single-configuration energy as a function of the two angles in the wave function. The left panel corresponds to $\delta=0.2$ and the right one $\delta=0.8$. Black areas are the lowest in energy. The proper symmetry of the wave function is found on the dashed diagonal line. The broken-symmetry energy minima of $\delta=0.2$ correspond to a single configuration, and for $\delta=0.8$ to SDW solutions.

energy of the mean-field state is equal to the average of the two exact energies. Furthermore, if one assumes that $\psi_{1}$ has a node on the $x$ axis and $\psi_{2}$ on the $y$ one, one can find densities similar to the SDW solution above. Now for the $\mathrm{QD}$, the value of $\delta$ is close to 0.8 , and one can understand the occurrence of the SDW solution from this more general argument.

The SDW solutions of Ref. 1 for larger particle numbers can equally well be understood based on the four-electron case and the general argument presented above. In the cases where two Kohn-Sham orbitals are degenerate, we have a $S=1$ ground state. When the aspect ratio $\beta$ is changed, the energies split and one always finds a broken-symmetry SDW solution. The similarity to the four-electron case follows from the fact that in all these cases, there are two spatial orbitals of both spin type occupied by two electrons. The spin density in the SDW structure can then be directly found from the two nearly degenerate states as $\left(\psi_{1}+\psi_{2}\right)^{2}-\left(\psi_{1}\right.$ $\left.-\psi_{2}\right)^{2}$. For example, the densities in Fig. 6 of Ref. 1 are accurately reproduced by this formula using for the degenerate states the noninteracting ones with quantum numbers $(1,3)$ and $(3,2)$ for the left panel, or $(3,2)$ and $(4,1)$ for the right panel.

\section{RECOVERING SYMMETRY}

Based on the results presented above, it is clear that standard DFT is not able to describe accurately E-VR systems. The method of Ullrich and $\mathrm{Kohn}^{3}$ is one possible solution, but this method might have an underlying problem. Namely, even in the case of an open shell and degeneracy, there are systems that still are P-VR, simply because the configurations do not necessarily mix even if they are degenerate. One such example is the parabolic QD, where the angular momentum is a good quantum number and single-particle states can be chosen in such a fashion that only one major configuration is found. It is not straightforward to see how the method of Ref. 3 assort the open-shell cases that are E-VR from those that are still P-VR.

As a possible solution we propose a scheme where first a standard DFT calculation is performed for the system (with- 
out symmetry breaking but with fractional occupations for the degenerate levels). For our case with $\beta=1$, the occupations of the two highest orbitals $\psi_{1}$ and $\psi_{2}$ are $1 / 2$ and the DFT energy is 13.26. One can construct two $S=S_{z}=0$ DFT configurations that have density equal to the DFT one by defining new orbitals $\phi_{ \pm}=\psi_{1} \pm i \psi_{2}$. Now the configurations involve the core DFT orbital $\psi_{0}$, and either $\phi_{+}$or $\phi_{-}$. The occupied orbitals in configurations are the same for both spin electrons, similarly to $C_{1}$ and $C_{2}$ in Fig. 1. The coupling of these two DFT configurations can be approximated by the basic formula by Slater ${ }^{9}$ as

$$
\delta=\int \phi_{+}^{*}\left(\mathbf{r}_{1}\right) \phi_{+}^{*}\left(\mathbf{r}_{2}\right) \frac{1}{r_{12}} \phi_{-}\left(\mathbf{r}_{1}\right) \phi_{-}\left(\mathbf{r}_{2}\right) d \mathbf{r}_{1} d \mathbf{r}_{2} .
$$

Now the DFT energy gives the diagonal Hamiltonian matrix elements and thus incorporates partly correlations, and the above formula for $\delta$ gives the off-diagonal Hamiltonian matrix elements that result from the multiconfigurational nature of the ground state. The two-ensemble DFT energy can be found by diagonalizing the Hamiltonian matrix. For our example, the absolute value of $\delta$ is found to be $\approx 0.14$. Thus the mixing of the two configurations lowers the DFT energy to 13.12. This value is consistent with the ED one, if one takes into account the difference in DFT and ED energies for the nondegenerate cases. One should note that for a parabolic $\mathrm{QD}$, the absolute value of $\delta$ is zero (resulting from the rotational symmetry), meaning that the configurations (with different angular momentum) do not mix. This also shows that our scheme correctly predicts the system to be P-VR. A similar behavior can be found for the multiplets in open-shell atoms.

The underlying idea of the scheme presented above is that DFT is able to efficiently describe correlation effects in a certain subspace of the full Hilbert space. This subspace is related to a one DFT configuration. In P-VR cases this is sufficient for the accurate description of the system, but for an E-VR case, there are two or more subspaces relevant for the ground state, and DFT is unable to couple these. This coupling can be introduced, and one natural way is via $\delta$ of Eq. (4) above.

The generalization of the scheme for cases without an exact degeneracy of the DFT orbitals is straightforward. In addition, the approximation made for $\delta$ can be directly used for cases with larger particle numbers, too. This is because the states that are occupied in both configurations do not appear in the formula for $\delta$. We believe that the presented approach shows to be useful for many applications of DFT, especially for molecules, where the calculations of chemical reactions have observed similar problems of basic DFT. ${ }^{10}$ More details and comparisons with other ensemble DFT approaches are left for forthcoming studies.

\section{CONCLUSIONS}

Concluding, we have shown that the use of a singleconfiguration wave function in a mean-field theory can lead to an unphysical solution with a broken symmetry. In our case of a four-electron rectangular QD, the energy of the SDW solution is reasonable, but the spin densities have only a minor similarity with the exact total or conditional ones. We also present an analysis with a more general Hamiltonian matrix and we feel that our findings are relevant for a great variety of systems studied by the mean-field approaches, DFT in particular. As a cure, we propose a scheme for incorporating systematically several configurations into a meanfield approach. The method presented avoids the necessity of symmetry breaking, and has a built-in criteria to determine if several configurations are actually needed or not.

\section{ACKNOWLEDGMENTS}

We thank R. van Leeuwen, V. Sverdlov, and E. Thuneberg for discussions and acknowledge support by the Academy of Finland's Centers of Excellence Program (2000-2005).
${ }^{1}$ E. Räsänen, H. Saarikoski, V.N. Stavrou, A. Harju, M.J. Puska, and R.M. Nieminen, Phys. Rev. B 67, 235307 (2003).

${ }^{2}$ S.M. Reimann and M. Manninen, Rev. Mod. Phys. 74, 1283 (2002).

${ }^{3}$ C.A. Ullrich and W. Kohn, Phys. Rev. Lett. 87, 093001 (2001).

${ }^{4}$ A.W. Overhauser, Phys. Rev. Lett. 4, 462 (1960); Phys. Rev. 128, 1437 (1962).

${ }^{5}$ G. Grüner, Rev. Mod. Phys. 66, 1 (1994).

\footnotetext{
${ }^{6}$ S. Frauendorf, Rev. Mod. Phys. 73, 463 (2001).

${ }^{7}$ H. Häkkinen, J. Kolehmainen, M. Koskinen, P.O. Lipas, and M. Manninen, Phys. Rev. Lett. 78, 1034 (1997).

${ }^{8}$ K. Hirose and N.S. Wingreen, Phys. Rev. B 59, 4604 (1999).

${ }^{9}$ J.C. Slater, Phys. Rev. 38, 1109 (1931).

${ }^{10}$ P.R.T. Schipper, O.V. Gritsenko, and E.J. Baerends, J. Chem. Phys. 111, 4056 (1999).
} 